(C2006 IEEE. Personal use of this material is permitted. However, permission to reprint/republish this material for advertising or promotional purposes or for creating new collective works for resale or redistribution to servers or lists, or to reuse any copyrighted component of this work in other works must be obtained from the IEEE. 


\title{
Trust Ontology for Service-Oriented Environment
}

\author{
Farookh Khadeer Hussain ${ }^{1}$, Elizabeth Chang $^{1}$ and Tharam S.Dillon ${ }^{2}$ \\ ${ }^{1}$ School of Information Systems \\ Curtin University of Technology \\ P.O. Box U1987 \\ Perth, Western Australia, Australia \\ \{Farookh.Hussain, Elizabeth.Chang\}@cbs.curtin.edu.au \\ ${ }^{2}$ Faculty of Information Technology \\ University of Technology, Sydney \\ Sydney, NSW 2007, Australia \\ tharam@it.uts.edu.au
}

\begin{abstract}
Trust and Reputation are vital components for trusted e-business. In the literature however there has been no effort in proposing ontology for trust.

The trusted agent in service oriented environment may trust a software agent or human agent or a service or a product. Based on this distinction, trust ontology could be proposed for different domains.The trust ontology for the individual domains is proposed and discussed.
\end{abstract}

\section{Introduction}

Ontology can be viewed as a shared conceptualization of a domain that is commonly agreed to by all parties. It is defined as 'a specification of a conceptualization' [2]. 'Conceptualization' refers to the understanding of the Concepts and relationships between the Concepts that can exist or do exist in a specific domain or a community. A representation of the shared knowledge in a specific domain that has been commonly agreed to refers to the 'specification' of a Conceptualization.

From the existing literature we note that there has been no effort to propose ontology for trust and reputation. In this paper we propose ontology for trust. This paper is organized as follows:

- In Section 2, we propose what we mean by Trustworthiness of Product and Trustworthiness of Service and Trustworthiness of Agent.

- In Section 3, we propose a hierarchy of the trust concepts.

- In Section 4, we propose an ontological representation of Agent Trust.
- In Section 5, we propose an ontological representation of Service Trust.

- In Section 6, we propose an ontological representation of Product Trust.

- Section 7 concludes the paper and explains the future work.

\section{Trustworthiness of Product, Service and Agent}

The trusting agent can develop trust in an agent, or product or service. Trust by a trusting agent could hence be directed to three distinct groups of entities, i.e. Agents, Products and Services. Trust between a Trusting Agent and Trusted Agent signifies the belief that the Trusting Agent has about the Trusted Agent's willingness and capability to deliver on the mutually agreed behaviour in a given context and time slot.

Trust by a trusting agent in a service, refers to the belief that the Trusting Agent has about the quality of Service. In other words, the Trusting Agent trusts that the quality of the service provided by the service provider will be as expected by the trusting agent. The expectation of the trusting agent about the quality of service is determined during the negotiation phase with the service provider. Interested readers are encouraged to refer to [1] for a detailed discussion and explanation on the negotiation phase.

Trust by a trusting agent in a product, refers to the belief that the Trusting Agent has about the Product's "Quality". In other words the Trusting Agent is of the belief that the quality of the product will be as it expects.

The expectation of the trusting agent about the quality of product is determined or derived from the product manual or the advertisement of the product that is 
provided by the product manufacturer. The manual of the product lays down the functionality and the specification of the product. The Trusting Agent can determine whether a given functionality could be accomplished or delivered to by a given product by going through the list of all functionalities listed in the product manual / product catalogue or the advertisement and finding out if the list of all the functionalities contains the functionality that the Trusting Agent is looking for in the product. Additionally the manual should quantify in quantitative terms how the functionality is delivered using the commonly used metrics to quantify the functionality. As an example, let us assume that a Bob likes the XLE-3400 Digital Camera manufactured because it has a resolution of 10 Mega Pixels. Furthermore let us assume that Bob decided to purchase the camera after reading the product manual of XLE-3400. The trust relationship for between Bob and the digital camera is specified to the resolution of the camera only. The resolution of the digital camera XLE-3400 is quantified numerically in commonly used and understandable metrics in the product manual.

Trust in a given product, could be developed by a given Trusting Agent by making use of $3^{\text {rd }}$ Party Recommendation Agents who have made use of the product. The functionality for which the trusting agent trusts the product can be regarded as the context of the trust relationship between the trusting agent and the product.

Additionally the strength of the trust relationship between the Trusting Agent and the product represents the quality of the product as perceived by the trusting agent. The quality of a given product in service-oriented environments is determined by determining the correlation between

The delivered value of the product (and)

(ii) The mutually agreed value of the product

The quality of a given service in service-oriented environments is determined by determining the correlation between

(a) The delivered quality of service (and)

(b) The mutually agreed quality of service.

The trustworthiness of an agent in service-oriented environments is determined by determining the correlation between

(1) The actual behaviour of the trusted agent in the interaction (and)
(2) The mutually agreed behaviour of the trusted agent in the interaction.

We define the actual behaviour of the trusted agent as the set of all the functionalities that the trusted agent has delivered to the trusted agent in the interaction.

We define the mutually agreed behaviour of the trusted agent as the set of all the functionalities that the trusted agent has agreed to deliver to the trusted agent in the interaction.

In this paper, we define the Trust Ontologies that are to be used in Service-Oriented Environments. In a ServiceOriented Environment, business entities or Agents can carry out buying and selling of services and goods, requesting or inquiry of information, biding or offering contracts, publishing or advertising of products [1]. As communication is between virtual entities who may or may not interacted with each other in the past, determining the Quality of a Service, the Quality of a Product or the Quality of a Trusted Agent is one of the biggest considerations of the on-line consumers. It is one of the major barriers to e-Commerce development and the adoption of e-Commerce. Therefore, the study of Trust and developing Trust technologies for the ServiceOriented Environment has a strong impact on consumers, businesses and the economic environment. If the Quality of e-Services, e-Product etc via the Internet is guaranteed, many more people will use it, and it will bring about a revolution in e-Commerce and push the networked economy to a new level.

In the Service-Oriented Environment, as pointed out in [1], the Trusting Agent can develop trust in an agent, or product or service, Correspondingly a Trust Ontology could represent at least one of three domains, namely:

- Agent Trust Ontology

- $\quad$ Service Trust Ontology

- $\quad$ Product Trust Ontology

This Paper will give a detailed explanation of each of the above Trust Ontologies and its application to specific Trust Ontologies.

\section{Hierarchy of Trust Concepts}

In a Service-Oriented Environment, Trust can be visualized in at least in three domains, namely, Agent Trust, Service Trust and Product Trust.

In the Figure 1, there is a box is named as "Trust". In the middle-upper level of the diagram, there is a list a number of generic Concepts, namely: Agent Trust, Service Trust 
and Product Trust. These Concepts shall commit (or confirm or inherit) the entire top layer Ontology and its property specification. The detailed Ontology for each of these domains is presented in the rest of this Paper.

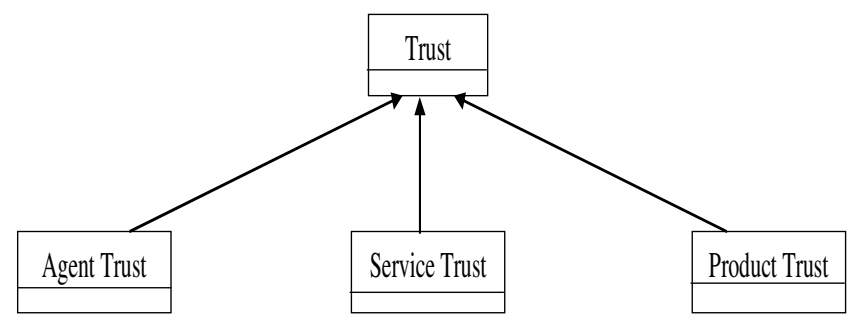

Figure 1: Generic Concept of Trust and its relationship to specific 'Agent Trust', 'Service Trust' and 'Product Trust' Concepts.

\section{Agent Trust Ontology}

As explained earlier, Trust in the Service-Oriented Environment can represent three domains, namely: Agent Trust, Service Trust and Product Trust. In this section, we propose and explain the Agent Trust Ontology.

Agent Trust Ontology: In a service-oriented network environment, the Generic Agent Trust Ontology is defined as the Conceptualization of the Agent Trust (that is, the trust that the Trusting Agent has in a given Trusted Agent in a given context and a given timeslot).

The graphical view of Generic Agent Trust Ontology is shown in Figure 2 through the use of the Ontology notation, which we have proposed in earlier publication [1]. Explaining the ontology notations is out side the scope of this paper. Interested readers are encouraged to refer to [1] for an explanation of the ontology notations.

We represent the Agent Trust Ontology as the combination of the Ontology name and a Tuple where the elements of the Tuple can be complex elements as defined below:

Agent Trust [Trusting Agent, Trusted Agent, Context, Criteria, Quality Assessment Metrics, Timeslot, and Trustworthiness Value] where:

- 'Trusting Agent' is the agent who has reposed trust in another agent.

- 'Trusted Agent' is the agent in whom trust has been reposed by the Trusting Agent.

- 'Context' is the scenario in which Trust has been reposed by the Trusting Agent in the Trusted Agent.

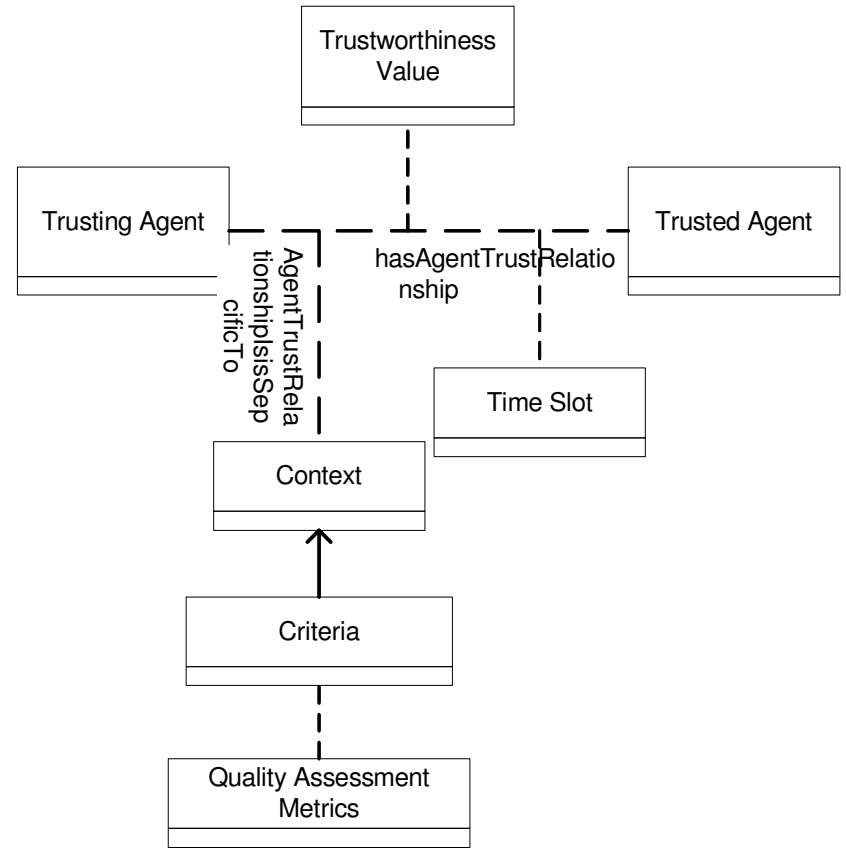

Figure 2 Ontology Representation of Agent Trust Concept and its relation to other Concepts

- 'Criteria' is used to refer to the individual criterion based on which the trusting agent is going to access and assign a trustworthiness value to the trusted agent. A criteria contains at least one criterion.

- 'Quality Assessment Metrics' (or) Criteria Assessment Metrics are metrics that are used to determine or assess whether or not a trusted agent has fulfilled a given criteria or not.

- 'Trustworthiness Value' quantifies and expresses the trust that the trusting agent has in the trusted agent. Once Trust has been quantified it could be represented on the Trustworthiness Scale.

- 'Timeslot' defines the duration of time during which the trustworthiness value between the trusting agent and the trusted agent is valid.

Agent Trust is measured by criteria that are mutually agreed by both the Trusting Agent and the Trusted Agent during the Negotiation Phase. In an earlier publication[1],we have explained how the trusting agent can determine the trustworthiness of the a given trusted agent based on the based on the criteria that were agreed by both the interacting parties during the Negotiation Phase, by making use of the Quality Assessment Metrics. 
Interested readers are encouraged to refer to [1] for an explanation of how the trusting agent can determine the trustworthiness value of the trusted agent by making use of CCCI Metrics.

Agent Trust is determined using the criteria that were agreed to by both the interacting parties during the Negotiation Phase. An important distinction to note here, is that the both the trusting agent and the trusted agent enter a Negotiation Phase, to develop a mutually agreed criteria. Based on the mutually agreed criteria and the quality assessment metrics, the trusting agent would assign a trustworthiness value to the trusted agent.

\section{Service Trust Ontology}

As explained in Section 1, Trust in Service-Oriented Environment can represent three domains, namely: Agent Trust, Service Trust and Product Trust. In this section we propose the service trust ontology.

Service Trust Ontology: In service-oriented network environments, the Service Trust Ontology is defined as the conceptualization of the Service Trust (that is the trust that signifies that the Quality of Service $(Q o S)$ provided by a given Service Provider will be in accordance with the mutually agreed QoS in the given service context and timeslot ).

We represent the Service Trust Ontology as the combination of the Ontology name and a Tuple where the elements of the Tuple can be complex elements as defined below:

Service Trust [Service Requester, Service, Criteria of Service, Quality Assessment Metrics, Time Slot, Service Provider, and Quality of Service $(Q o S)]$ where:

- 'Service Requester' is the Trusting Agent who reposed trust in a given service.

- 'Service Provider' is the Trusted Agent who provides the service to the Service Requestor.

- 'Service' is the scenario in which trust has been reposed by the trusting agent in the service provided by the Service Provider. It refers to the type of service provided by the service provider. Service represents the context of the trust relationship between the Service Requestor and the Service Provider. In other words, service is used to represent the set of all coherent related functionalities that the trusting agent is looking for in its interaction with the trusted agent.

- 'Criteria of Service' refers to the various conditions or aspects of the service that were agreed to by the Trusting Agent and the Service Provider during the negotiation phase. The various criteria of the service are documented in the service agreement. A service is comprised of one or more criteria of service.

In other words 'criteria of service' is used to represent the individual functionality that the trusting agent is looking for in its interaction with the trusted agent.

- 'Quality Assessment Metrics' or 'Criteria Assessment Metrics' refers to the policies or the metrics based on which the service requestor is going to access the extent to which a given criteria of service has been is fulfilled by the service provider.

- 'QoS' is the measure that signifies that the quality of the service provided by the Service Provider for the service.

- 'Timeslot' defines the duration of time during which the trustworthiness value between the Service Requestor and the Service Provider is valid.

Service Trust is measured by criteria that are mutually agreed by both the Service Requestor and the Service Provider the Negotiation Phase. Similar to Agent Trust, Service Trust is determined using the criteria that were agreed to by both the interacting parties during the Negotiation Phase. An important point to note here is that similar to the Agent Trust, both the service provider and the service requestor enter a Negotiation Phase, to develop mutually agreed criteria. Based on the mutually agreed criteria and the quality assessment metrics, the service requestor would determine the Quality of Service (QoS) provided by the service provider.

The ontological representation of service trust is shown in Figure 3, at the end of the paper.

\section{Product Trust Ontology}

As explained earlier, Trust in a Service-Oriented Environment can represent three domains, namely: Agent Trust, Service Trust and Product Trust. In this section, we describe the Product Trust Ontology.

We often evaluate products we buy, whether they are worth the expenditure, or whether they are of good or bad quality. Positive evaluations attract us to buy more or make recommendations to acquaintances to buy more. When we evaluate products, it is based purely on our opinion, as a 'product' is unable to respond if we give unfair opinions. This is different than providing opinions about Agents or Service Providers, because the judgment has to take both parties' opinion into consideration. This 
sometimes involves witnesses or third parties in order to make fair judgments.

The Ontology for Product Trust is similar to the ontology for Agent Trust. However, the similarity is at the hierarchy level only and not at the semantic level.

Similar to the measurement of the 'Quality of Agent (QoA)' and the 'Quality of Service (QoS)' the Quality of Product (QoP) is determined by measurement against a set of criteria that are pertinent to the specific class of product/s whose trustworthiness is being measured.

In Service-Oriented Network environments, the Product Trust Ontology is defined as the conceptualization of the Trust that the customer has in a product and its quality aspects defined in the product specification and satisfactory fulfillment of all the Assessment Criteria in a given timeslot.

We represent the Product Trust Ontology as the combination of the Ontology name and a tuple where the elements of the tuple can be complex elements as defined below:

Product Trust [Buyer, Product, Context of Product, Criteria of Product, Quality Assessment Metrics, Time Slot, and QoP Measure] where:

- 'Buyer' is a Trusting Agent who is purchasing the product and will subsequently evaluate the product and determine the quality of the product (QoP).

- 'Product' is the Trusted Entity or object which is being purchased by the Trusting Agent and whose quality is being accessed by the buyers.

- 'Context' is the term that is used to jointly refer to coherent functionalities that the trusting agent is looking for in its interaction with the trusted agent.

- 'Criteria' is used to refer to the individual functionality that the trusting agent is looking for in its interaction with the trusted agent.

- 'Quality Assessment Criteria' or 'Criteria Assessment Factors' are the factors that the trusting agent would use to determine whether or not a specific criterion was delivered by the product.

- 'Timeslot' is the timeframe for which the Trust Value holds, i.e. During this period, the Trust Value remains the same.

- 'QoP Measure' expresses the quality of the product on the Trustworthiness Scale.

The graphical view of Product Trust Ontology is shown in Figure 4, through the use of the Ontology Notation.

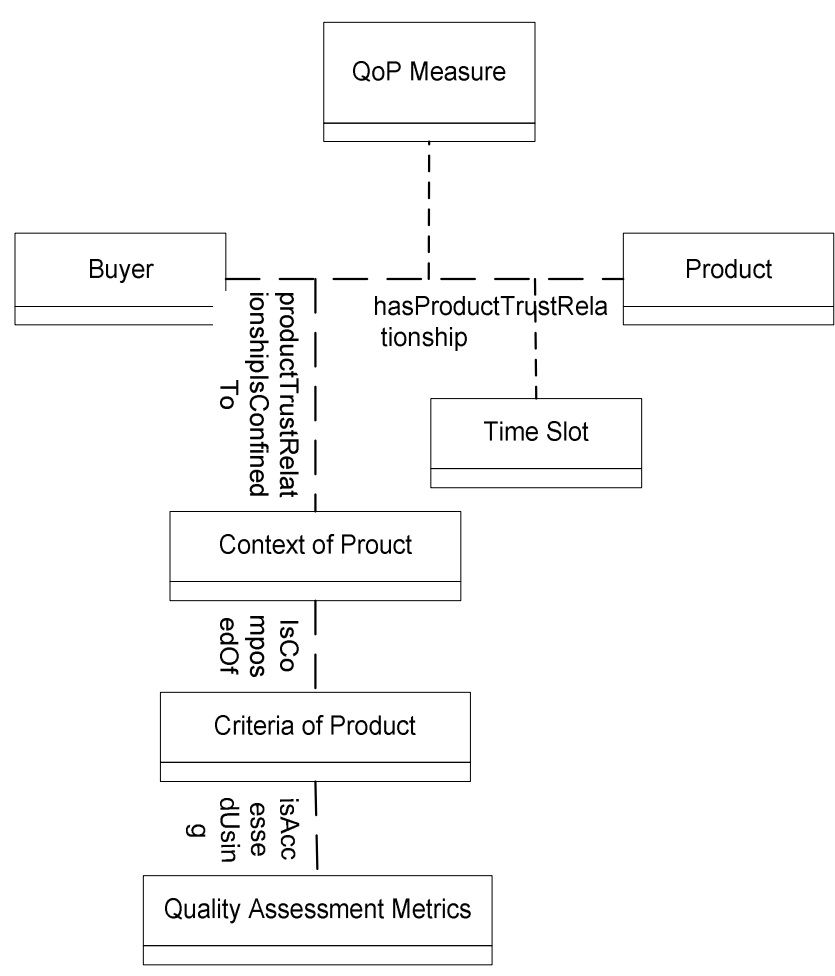

Figure 4. Ontology Representation of Product Trust Concept and its relation to other Concepts

\section{Conclusion}

We found that trust in service oriented environments could be directed towards either a agent (software agent or human agent), product or service. In this paper we proposed an ontological representation of agent trust, service trust and product trust.

Our further work involves validating the proposed trust ontology and proposing an ontological representation of reputation in service oriented environment.

\section{References}

[1]Chang, E., Dillon, T.S., Hussain, F.K., 'Trust and Reputation for Service Oriented Environment', John Wiley and Sons (To be published in 2005).

[2] Gruber, T.R., (1993), 'A Translation Approach to Portable Ontologies', Knowledge Acquisition, Vol.5 (2) pp. 199-220. 


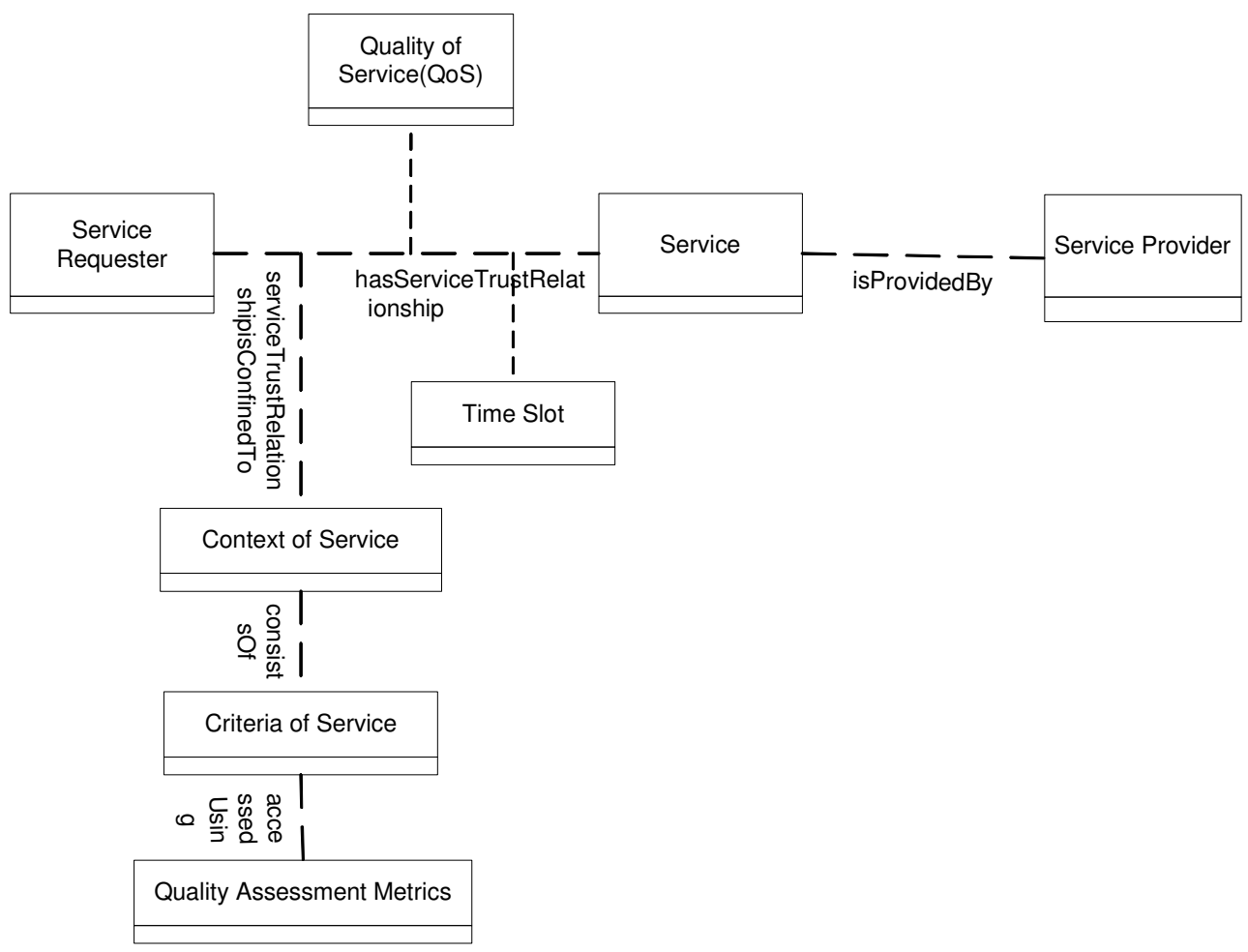

Figure 3. Ontology Representation of Service Trust Concept and its relation to other Concepts 